\title{
Chromospherically Active Stars in the RAVE Survey
}

\section{M. Žerjal ${ }^{1}$, T. Zwitter ${ }^{1,2}$, G. Matijevič ${ }^{3}$, K. G. Strassmeier ${ }^{4}$ and RAVE Collaboration}

${ }^{1}$ Faculty of Mathematics and Physics, University of Ljubljana, Jadranska 19, 1000 Ljubljana, Slovenia

email: marusa.zerjal@fmf.uni-lj.si

${ }^{2}$ Center of Excellence SPACE-SI, Aškerčeva cesta 12, 1000 Ljubljana, Slovenia

${ }^{3}$ Dept. of Astronomy and Astrophysics, Villanova University, 800 E Lancaster Ave, Villanova, PA 19085, USA

${ }^{4}$ Leibniz-Institut für Astrophysik Potsdam (AIP), An der Sternwarte 16, D-14482, Potsdam, Germany

\begin{abstract}
We present a qualitative characterization of activity levels of a large database of $\sim 44,000$ candidate RAVE stars (unbiased, magnitude limited medium resolution survey) that show chromospheric emission in the Ca II infrared triplet and this vastly enlarges previously known samples. Our main motivation to study these stars is the anti-correlation of chromospheric activity and stellar ages that could be calibrated using stellar clusters with known ages. Locally linear embedding used for a morphological classification of spectra revealed 53,347 cases with a suggested emission component in the calcium lines. We analyzed a subsample of $\sim 44,000$ stars with $S / N>20$ using a spectral subtraction technique where observed reference spectra of inactive stars were used as templates instead of synthetic ones. Both the equivalent width of the excess emission for each calcium line and their sum is derived for all candidate active stars with no respect to the origin of their emission flux. $\sim 17,800$ spectra show a detectable chromospheric flux with at least $2 \sigma$ confidence level. The overall distribution of activity levels shows a bimodal shape, with the first peak coinciding with inactive stars and the second with the pre-main-sequence cases.
\end{abstract}

Keywords. stars: chromospheres, activity

\section{Introduction}

RAdial Velocity Experiment (RAVE, Steinmetz et al. (2006), Zwitter et al. (2008), Siebert et al. (2011) and Kordopatis et al.(2013) in preparation) is an unbiased magnitude limited $(9<I<12)$ spectroscopic southern sky survey that measured over 570,000 spectra of 480,000 stars. The observing campaign lasted for ten years from 2003 to 2013. The aim of the survey was a determination of radial velocities and an estimation of effective temperature, surface gravity and metallicity of Galactic stellar populations. All spectra cover the near-infrared range from $\sim 8410$ to $\sim 8795 \AA$ where the Ca II triplet $(\lambda \lambda=8498,8542,8662 \AA)$ dominates the spectral shape on a broad range of atmospheric parameters. The typical resolving power of the spectra is $R \sim 7500$; their typical $S / N$ is $\sim 40$.

Matijevič et al. (2012) classified a subsample of $\sim 350,000$ spectra with the locally linear embedding method (LLE) and showed that $~ 90 \%-95 \%$ of spectra belong to normal single stars. However, two large distinct groups of peculiars were found. One of which is active stars that show chromospheric emission in calcium lines ( 50,000 stars).

The chromospheric emission is seen as an additional flux at the central wavelengths of 


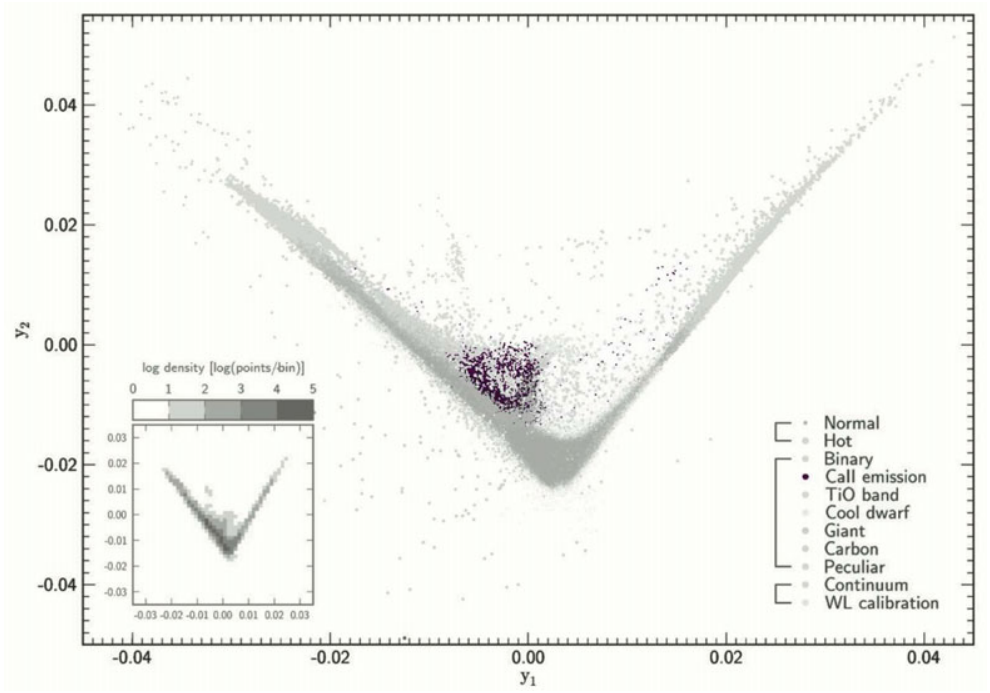

Figure 1. Locally linear embedding was used to explore the morphology of RAVE spectra. Stars with similar morphological features lie in the same area of the plot. Candidates for active stars show as a bump - black dots that emerge upwards nearly from the middle of the ' $v$ '-shape. The bottom inset shows the log density of points on the main diagram. For color version of the plot see the original paper by Matijevič et al. (2012).

strong lines. It occurs in young main sequence solar-type or cooler dwarfs with convective envelopes and affects strong spectral lines with the largest impact on $\mathrm{H} \alpha$, Ca II $\mathrm{H} \& \mathrm{~K}$ (3969, $3934 \AA$ ) and the Ca II infrared triplet (IRT). The most interesting phenomenon, among others, is the fact that the chromospheric activity level correlates with a diminishing stellar rotation rate through the coupling by the magnetic dynamo effect and can therefore be used as an age diagnostics tool for the nearest relatively young stars (0.6-4.5 Gyr, Mamajek \& Hillenbrand (2008)).

The majority of existing emission data-sets in the literature cover the Ca II H \& K lines. However, Žerjal et al. (2013) report a sample of newly discovered $\sim 44,000$ candidate active stars in the Ca II IRT of the RAVE survey. These candidate active stars cover a wide range of activity levels from very strong emission objects to numerous examples with only a marginal detection or no detection at all. Although the emission in most of the spectra comes from the chromospheres, the presence of an emission component in some rare cases does not necessarily imply a chromospheric origin, due to the selection criteria when building the catalogue of candidate active stars. For example Strassmeier et al. (2005) report the emission component in classical T Tauri stars where the emission partly comes from accretion shocks. For this reason the term 'active star' refers to a candidate active star within this paper.

The following chapters briefly summarize the measuring technique for quantitative description of the activity levels from the RAVE catalogue of candidate active stars. A detailed description is given in Žerjal et al. (2013, submitted).

\section{A sample of candidate active stars}

The selection criteria for stars from RAVE's database of 570,000 spectra to be recognized as a candidate active stars are based on the classification by the LLE method.

LLE is a general dimensionality reduction algorithm with a property of conserving locally linear patches of the high-dimensional manifold. The method is executed in three 

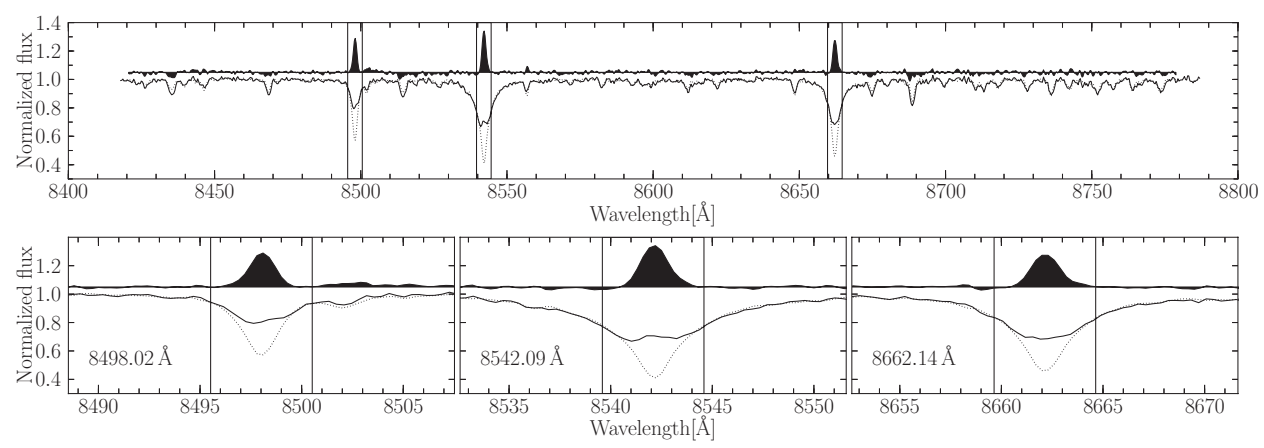

Figure 2. Top panel: An example for the RAVE spectrum of a candidate active star. The solid line is the spectrum itself and the dotted line is its nearest normal neighbour that is used to determine the subtracted spectrum (black area on the top). The three bands are the $5 \AA$ wavelength ranges that are not taken into account when searching for the nearest neighbour but are used in determining stellar activity rate. Bottom panels: Enlarged sections with calcium lines. Central wavelengths for each line are given at the left bottom corner.

separate steps. First, it finds the nearest neighbours for each data point. Then, it calculates the weights that best represent a given data point as a linear combination of its neighbours in the original space. Finally, it finds a low-dimensional space in which each data point is still best represented by the same set of weights that were calculated in the previous step. For a more detailed description of the method see Roweis \& Saul (2000), Vanderplas \& Connolly (2009) or Matijevič et al. (2012) and references therein. Matijevič et al. (2012) extended the method for spectral classification purposes. Their result (see Fig. 1) is a database where every spectrum contains morphological information of its 20 nearest neighbours in the template database of 5000 manually classified spectra that are sufficiently distributed over the whole morphological space. More details are given in their paper itself.

Our data-set of $\sim 44,000$ candidate active stars with $S / N>20$ includes all objects from the RAVE database that have at least one chromospherically active neighbour within its first 6 neighbours. That constraint is very loose and doesn't take into account the possibility that other neighbours can be peculiar.

\section{Estimation of chromospheric activity level}

One of the most widespread proxies for Ca II H \& $\mathrm{K}$ chromospheric activity is the dimensionless $\mathrm{R}_{\mathrm{HK}}^{\prime}$ (Noyes et al. (1984)). It is derived from the equivalent width of the emission flux which is revealed by removing the photospheric contribution from the active spectrum. In practice this is done by subtracting the synthetic inactive counterpart from the active spectrum. The remaining spectrum represents the chromospheric emission flux.

We seized the opportunity of the extremely large RAVE database of normal stars and used observed non-peculiar spectra instead of synthetic templates to compute the subtracted spectra. We built a database of 32,787 normal stars that covers the whole parameter space (effective temperature, metallicity and gravity variations, possible variations of resolving power along the spectrum and rotational velocity). The term 'normal spectra' is used to denote single lined stars that show no peculiarities. A spectrum was recognized as normal in the analogous manner as in the case of the selection of active stars. The normal database was built with additional constraints: $S / N>30$ and the spectra must have temperatures below $7000 \mathrm{~K}$. The lower limit in the temperature is 
$3500 \mathrm{~K}$ because this is the limit of the synthetic grid (Munari et al. 2005) used in the RAVE DR3 pipeline (Siebert et al. 2011).

The best matching templates from the normal database for each of the active spectra are found by the $\chi^{2}$ nearest neighbour search where the cores of the calcium lines are not taken into account. We removed three $5 \AA$-wide ranges $( \pm 2.5 \AA$ around the central wavelength of each calcium line). The width of the cut was determined by visual inspection of the most active spectra where the emission peak is the most prominent and hence the widest (see Fig. 2). Quite numerous cases of moderate velocity shifts of the emission components were another reason for such wide line removals.

All RAVE spectra were continuum normalized by the RAVE pipeline and shifted to zero heliocentric radial velocity. While radial velocities of normal stars are highly reliable, emission components in calcium lines could affect parameter and velocity determination. For this reason the nearest neighbour search was performed iteratively: First step of each iteration was search for the best matching template. Next, the active spectrum was shifted to zero velocity with respect to that template. Since calcium lines were missing, relatively strong Fe I spectral lines $(8674.75,8688.63 \AA$ ) were used to compute the crosscorrelation function and to perform the velocity shifts. Then we returned to step number one and repeated the loop until the last velocity correction decreased below $1 \mathrm{~km} \mathrm{~s}^{-1}$ (pixel width is $10.5 \mathrm{~km} \mathrm{~s}^{-1}$ ).

\section{Activity}

After the templates for each of the active spectra were found, we removed their photospheric contribution by subtracting them. The equivalent widths $\left(\mathrm{EW}_{\lambda}\right)$ of emission flux of each calcium line were measured first from the subtracted spectra on $5 \AA$ intervals $( \pm 2.5 \AA$ around the central wavelengths). The equivalent widths for each line in each spectrum were derived five times using the first five nearest templates, because it turned out that the average $\mathrm{EW}_{\lambda}$ computed with the first 5 nearest neighbours was not significantly different from an average using 10, 15 or 20 nearest neighbours. The stability of $\mathrm{EW}_{\lambda}$ confirms the assumption that our normal database completely and sufficiently covers the whole parameter space. Errors (the scatter) of $\mathrm{EW}_{\lambda}$ were estimated as the standard deviation of activity levels within 5 neighbours; typical uncertainties on the equivalent width of the emission component of each calcium line are $0.05 \AA$.

The equivalent widths of the chromospheric flux for each particular line mostly range from $\sim-0.2 \AA$ to $\sim 1 \AA$. Most active stars show even larger values, while noise and template mismatch can be a reason for negative $\mathrm{EW}_{\lambda}$. Tight correlation of line strengths confirms the consistency of the results and excludes the possibility that the emission signal comes from another source like cosmic rays or similar errors.

We define the term 'activity' as a sum of equivalent widths for all three calcium lines for each star:

$$
\mathrm{EW}_{\mathrm{IRT}}=\mathrm{EW}_{8498}+\mathrm{EW}_{8542}+\mathrm{EW}_{8662} .
$$

The overall distribution of activity $\left(\mathrm{EW}_{\mathrm{IRT}}\right)$ is shown in Fig. 3 and clearly shows a bimodal distribution with two peaks at $\sim 0.1$ and $\sim 1.5 \AA$.

For reference the activity of normal stars was derived using the same method (we took 2nd-6th nearest neighbours since the first was the spectrum itself). The distribution of activity of the normal database is centered around $-0.01 \AA$ with a standard deviation of $\sigma=0.17 \AA$. This value is adopted as a general uncertainty in any EW IRT value for both normal and active stars. We searched for RAVE pre-main sequence stars that are recognized as such in the Simbad database; their distribution overlaps with the second 


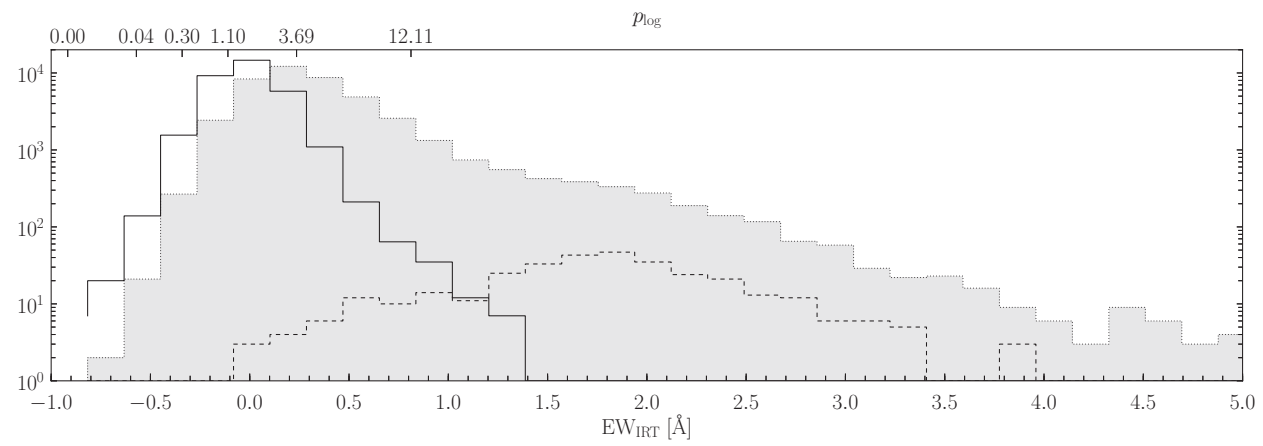

Figure 3. Distribution of EW $\mathrm{EWT}_{\mathrm{IRT}}$ for active stars (grey area), normal stars (which are assumed to be inactive; solid line) and pre-main sequence stars (selection is based on Simbad classification of RAVE stars; dashed line). The scale is logarithmic.

peak of active stars and perhaps implies a discovery of pre-main sequence stars in the RAVE survey.

\section{Discussion and conclusions}

The catalogue of candidate active RAVE stars that significantly enlarges previously known samples and is the topic of this summary is quantitatively described by Žerjal et al. (2013) in detail. It will become public and be part of the next RAVE data release (DR4). The selection criteria for the database of candidate active normal stars is based on the LLE classification of stellar spectra (Matijevič et al. 2012). Most of the stars from the active database that show additional emission flux are chromospherically active, but since it contains candidate stars with no respect to the origin of the emission flux, our results should be threated with caution. The estimation for activity levels is independent of the RAVE pipeline version because our method is based on a direct comparison of fluxes and omits the use of stellar parameters.

A spectral subtraction technique, with a large database of normal spectra (high $\mathrm{S} / \mathrm{N}$ single-lined stars without any peculiarities) as templates, was used to extract the equivalent widths of emission flux in Ca II IRT lines. The search for the best matching templates was performed iteratively because previously shifted candidate active stars (to the zero heliocentric radial velocity) still suffered from non-zero radial velocities due to their shallower calcium lines and improper treatment in the RAVE pipeline. For the same reason also the stellar parameters (effective temperature, metallicity and gravity) were re-estimated and are reported in our catalogue.

A distribution of stellar activity rates $\mathrm{EW}_{\mathrm{IRT}}$ that is shown in Fig. 3 has a clear bimodal appearance. The most populated peak are inactive and marginally active stars while the most active peak matches the position of pre-main sequence stars.

The comparison of our EW $\mathrm{ERT}_{\mathrm{IRT}}$ and $\log \mathrm{R}_{\mathrm{HK}}^{\prime}$ (for stars from 21 different catalogues from the literature) is shown in Fig. 4. Most stars are scattered around the less active area because these are the most abundant stars in our sample and the probability to find such a star in a reference catalogue is the highest. However, the comparison shows a good correlation between Ca II H \& K and our Ca II IRT activity levels but activities with $\log \mathrm{R}_{\mathrm{HK}}^{\prime}$ less than -5 are undetectable in the RAVE survey by our measurement technique.

This catalogue gives us an opportunity to further study the possible parameter dependence of activity and other phenomena, such as line asymmetries and Doppler shifts 


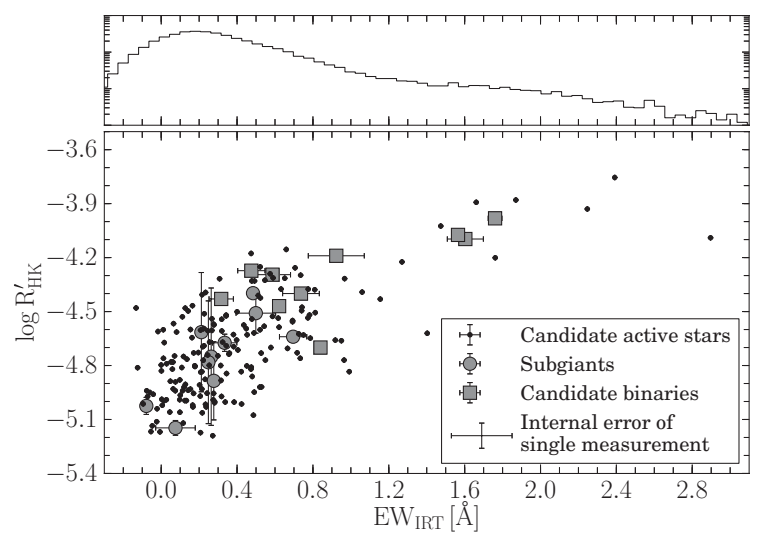

Figure 4. $\log \mathrm{R}_{\mathrm{HK}}^{\prime}$ versus $\mathrm{EW}_{\mathrm{IRT}}$ values for 211 RAVE stars matched with a set of available online catalogues. Some of the stars were measured multiple times by various authors or by RAVE; in this case we plot an average value for the star. The typical error of EW IRT is $0.17 \AA$. Our estimation of the $\log \mathrm{R}_{\mathrm{HK}}^{\prime}$ scatter is 0.05 . The size of typical internal error of single measurements is given in a legend. The histogram in the top panel shows the distribution of $\mathrm{EW}_{\mathrm{IRT}}$ for the whole sample of active RAVE stars in logarithmic scale.

of emission components. Since chromospheric emission anti-correlates with stellar ages (e.g. Soderlom et al. 2010), the most interesting application would be the age calibration using globular clusters and further study of the spatial distribution, the distribution with distances from Galactic plane as well as the kinematics of active stars in our Galaxy.

Since the RAVE catalogue of active stars consists of a relatively large number of objects it will be a suitable and convenient database to be compared with the results of the upcoming Gaia space mission (it covers Ca II IRT; Katz et al. (2004), Wilkinson et al. (2005)) and Hermes-Galah (which covers $\mathrm{H} \alpha$ and $\mathrm{H} \beta$; Zucker et al. (2012)).

\section{References}

Katz, D., Munari, U., Cropper, M., et al. 2004, MNRAS, 354, 1223

Mamajek, E. E., \& Hillenbrand, L. A. 2008, ApJ, 687, 1264

Matijevič, G., Zwitter, T., Bienaymé, O., et al. 2012, ApJS, 200, 14

Munari, U., Sordo, R., Castelli, F., \& Zwitter, T. 2005, A\&A, 442, 1127

Noyes, R. W., Hartmann, L. W., Baliunas, S. L., Duncan, D. K., \& Vaughan, A. H. 1984, ApJ, 279,763

Roweis, S. T. \& Saul, L. K. 2000, Science, 190, 2323

Siebert, A., Williams, M. E. K., Siviero, A., et al. 2011, AJ, 141, 187

Soderblom, D. R. 2010, ARA $\& A, 48,581$

Steinmetz, M., Zwitter, T., Siebert, A., et al. 2006, AJ, 132, 1645

Strassmeier, K. G., Rice, J. B., Ritter, A., et al. 2005, A\& A, 440, 1105

Vanderplas, J. \& Connolly, A. 2009, AJ, 138, 1365

Wilkinson, M. I., Vallenari, A., Turon, C., et al. 2005, MNRAS, 359, 1306

Žerjal, M., Zwitter, T., Matijevič, G., Strassmeier, K. G. et al. 2013, submitted

Zucker, D. B., de Silva, G., Freeman, K., Bland-Hawthorn, J., \& Hermes Team 2012, Galactic Archaeology: Near-Field Cosmology and the Formation of the Milky Way, 458, 421

Zwitter, T., Siebert, A., Munari, U., et al. 2008, AJ, 136, 421 